\title{
Directional Heterogeneity in Distance Profiles in Hedonic Property Value Models
}

by

Trudy Ann Cameron, University of Oregon

JEL Classifications: Q25, R21, H23

Correspondence: Department of Economics 435 PLC, 1285 University of Oregon, Eugene, OR 97403-1285 cameron@uoregon.edu

Acknowledgments: Research support accompanying the R.F. Mikesell Chair in Environmental and Resource Economics at the University of Oregon is gratefully acknowledged. Helpful suggestions and comments have been provided by J.R. DeShazo, Ryan Bosworth, Graham Crawford, Joe Stone, and participants at the AERE Annual Workshop in Madison, Wisconsin in June, 2003. 


\title{
Directional Heterogeneity in Distance Profiles in Hedonic Property Value Models
}

\begin{abstract}
Failure to allow for directional heterogeneity can obscure otherwise statistically significant distance effects in hedonic property value models. If ambient pollution data are unavailable, researchers often rely upon distance from a point source of pollution as a proxy for ambient environmental quality. However, damages from all types of point-source disamenities may exhibit directional heterogeneity. We generalize conventional distance models to allow for directional effects and show that commonly used linear and quadratic spatial trend variables capture directional heterogeneity in a manner that has not previously been recognized. Appropriate spatial models can also inform the social planner's problem of optimal allocation of source reduction across polluters. When independently calibrated tranport functions are not available, individual properties can be viewed as ambient receptor sites. Hedonic models can yield estimates of the product of marginal social damages from ambient concentrations and the change in ambient concentration per unit of emissions from each source. Optimal emissions depend upon the spatial distribution of all affected properties relative to each source, the parameters of the hedonic model, and marginal abatement costs.
\end{abstract}




\section{Introduction}

In some hedonic property value (HPV) models, researchers are able to quantify and use objective measures of localized environmental quality as specific attributes of each property in their sample. In other cases, however, it is more difficult to obtain objective measures of the level of an environmental attribute. When objective measures are unavailable or incomplete, researchers often resort to using distance from the source of pollution as a measurable characteristic. In this paper, the importance of testing for directional heterogeneity in distance effects is explored. We identify a number of different modeling strategies that can accommodate this heterogeneity, should it be present.

There have been a number of very competent general reviews of hedonic property value (HPV) models (for example, see Bartik and Smith (1987), Palmquist (1991), Freeman (1993), and most recently, Palmquist (2003)). Farber (1998) provides a useful inventory of empirical studies, including many that estimate distance gradients in housing prices. In particular, Michaels and Smith (1990) and Kohlhase (1991) find that distances from Superfund sites in Boston and Houston have a positive effect on house prices. The suite of papers by Kiel and her coauthors all control for distance to Superfund sites or hazardous waste incinerators and focus on a number different sites in Massachusetts (Kiel (1995), Kiel and McClain (1995), Kiel and Zabel (2001)). Dale, et al. (1999) emphasize housing prices over time as a function of distance from a lead smelter in Dallas, and McMillen and Thorsnes (2003) study the property value effects of the cleanup of a copper smelter site in Tacoma.

Hedonic models that do not allow for directional heterogeneity in distance effects assume that a twodimensional price-distance profile in a single quadrant is adequate to capture distance effects on property values. We can extend this distance profile to three dimensions by rotating it around the vertial axis. This leads to a price-distance surface that has circular level curves defining the depression in property values nearest the source of pollution. But distance effects may not be the same in all directions. These level curves

may be asymmetric around the pollution source. For example, many point sources of pollution produce 
either noticeable odors or airborne "fallout" so that prevailing winds can create directional asymmetries in distance effects. Industrial hog farming is an important regional example that has been addressed in the HPV literature by Palmquist, et al. (1997). However, the level curves for distance effects may also be asymmetric for other types of pollutants. The movement of surface or groundwater can propagate the damages from water pollution farther in some directions than others, and visual and noise pollution can be affected by topography.

We demonstrate in this paper that if substantial directional effects are present, but are ignored, one would expect a model with simple homogeneous distance effects to exhibit larger than necessary standard errors and apparent heteroscedasticity. Parameters defining the shape of the distance profile can also be biased if the directional distribution of distances for observed property transactions is non-uniform. We explore a number of models that can be used to test for the presence of directional heterogeneity in distance effects and can accommodate a wide variety of systematic directional effects when they are present.

A second important theme in this paper concerns an insight for the social planner's problem of optimal allocation of abatement responsibility across multiple point sources of non-uniformly mixing pollutants. Emissions transport and spatial heterogeneity in ambient environmental quality have long been recognized as important issues in the theory of pollution control policy. However, the systems of dedicated ambient monitors needed to calibrate the required "transport" functions are often incomplete so that the information embodied in these these key transport functions is unavailable to policy makers. We show how hedonic property value models with rich enough spatial differentiation in distance effects can yield estimates of the product of marginal damages and marginal transport effects. If marginal abatement costs at each source can be quantified, the estimated hedonic property value function and the spatial distribution of all properties affected by each source of emissions can be combined to suggest socially optimal emissions reductions for each source.

Published hedonic property value models that employ distance measures pay no attention to direction. 
Some authors use general spatial trend variables, either linear or quadratic. These researchers have implicitly allowed for directional effects but have generally failed to recognize that these trend variables contain key information about directional distance effects that cannot be ignored. There appear to be only two explicit considerations of direction in any form in the published hedonic literature. Gillen, et al. (2001) relegate directional considerations to the nature of spatial autocorrelation in the error terms in their model of isotropic versus anisotropic error autocorrelation in house prices. ${ }^{1}$ In their study of the effects of industrial hog-farming operations on house prices, Palmquist, et al. (1997) mention the problem of prevailing winds as an area for future research. However, confidentiality of specific locational data concerning hog farms prevented them from pursuing these issues. While prevailing winds would seem to be an important consideration in work concerning the "aroma of Tacoma" by McMillen and Thorsnes (2003), they do not consider direction either.

Section 2 of this paper explains why direction, as well as distance, may be an important feature of many HPV models. Section 3 outlines how commonly used spatial trend variables implicitly contribute to directional heterogeneity in distance profiles. Prevailing winds or other physical processes are likely to be a common denominator in cases where there is directional heterogeneity in distance profiles. Thus, section 4 reviews how external information about the annual average or seasonal directions of prevailing winds or other physical processes can be incorporated into the model. Section 5 outlines how the level curves for a directionally differentiated distance profile can be mapped. Section 6 pulls together the intuition from directionally heterogeneous distance profiles for housing prices and the social planner's problem of optimal allocation of pollution abatement responsibility among multiple sources of non-uniformly mixing pollutants. Section 7 provides some caveats before Section 8 concludes.

\footnotetext{
${ }^{1}$ There is no point-source environmental disamenity in their data from which distances are being measured; the only distances in the model are the distances between individual houses in the sample.
} 


\section{Distance Profiles as a Function of Direction}

Ignoring heterogeneity in distance effects with respect to direction from a localized pollution externality can potentially obscure what might otherwise be a clear price-distance relationship. Figure 1 illustrates just two different directions, East and West, rather than the full $360^{\circ}$ of the compass. In this case, the observations for the dependent variable, $Y_{i}$, are shown lying very close to the directionally-specific $E\left[Y_{i}\right]$. Each of these two directional distance profiles is depicted as a linear function of distance, $d$, with a common intercept, $\alpha$, but different slopes. ${ }^{2}$

Figure 1 is drawn under the assumption that prevailing winds are from the west. If the researcher controlled for direction before estimating the parameters of the distance gradient, the data in the example would yield very precise estimates of the common intercept, $\alpha$, and two separate slopes, $\beta_{E}$ and $\beta_{W}$. The steeper profile to the west indicates that the prevailing winds limit the westward diffusion of the pollutant. In contrast, the flatter profile to the east captures the fact that prevailing winds carry the pollutant considerably farther in that direction. Ignoring direction is equivalent to superimposing the two different distance profiles in the right-hand quadrant of the diagram and fitting one common distance profile to the pooled data. We illustrate the effect of ignoring direction by showing the western distance profile rotated around the vertical axis. The more heterogeneous the directional distance profiles, the greater will be the dispersion around the common "average" distance profile when direction is ignored. A second artifact of failure to control for direction will be apparent heteroscedasticity, with error variances increasing with distance.

In hedonic property value studies, researchers typically consider the "extent of the market" for proximity effects to consist of all housing transactions within a particular radius of an environmental hazard. Alternatively, the relevant market may consist of all census tracts or zip codes within a particular absolute distance from the site. In these cases, it may be approximately true that the distribution of distances at which property transactions are observed is independent of direction. When distance and direction are uncorrelated

\footnotetext{
${ }^{2}$ Contrast this form of heterogeneity with the type commonly assumed in fixed effects models for panel data. There, we typically assume a common slope, but different intercepts across groups.
} 
in the data, omitted variables bias from failure to control for direction will be minimized. However, there are a number of ways in which hedonic property value data may exhibit correlations between direction and distance. We will review these in turn.

In Figure 2, we expand the right-hand quadrant of Figure 1 to include an additional set of points (represented by open dots) for the intermediate north and south distance profiles. These north and south profiles are assumed to be identical since the prevailing winds are coming from due west. Suppose the researcher recognizes the potentially greater influence of pollution downwind, to the east, and therefore collects data to a greater distance in that direction. Figure 2 shows that if she fails to control for direction in the estimation process, it is possible that she may find no statistically significant relationship at all between $Y$ and distance, despite precise relationships in each direction considered separately. The empirical estimates could suggest a negligible or statistically insignificant distance effect-a "false negative"-when in fact distance effects are substantial and would be easy to discern when controlling for direction. The omitted variables bias in this case stems from the presence in the data of observation at greater distances only for a subset of all possible directions.

Figure 3 shows another potential source of omitted variables bias when direction is omitted. Even if the researcher collects data on $Y$ within a constant radius, regardless of prevailing winds, it is possible that observations are not naturally identically distributed with respect to distance in all directions from the Superfund site. This is a particular concern with data such as housing transaction price information. In the downwind direction, in the presence of a localized environmental externality, there may simply be fewer nearby houses. Alternately, there may be fewer nearby transactions if more-affected properties are more difficult to sell. If the nearest distance at which observations occur is greater in the downwind direction, slope distortions may result when direction is omitted from the model.

A third way in which correlations between distance and direction may naturally be present in hedonic property value data is when features of the landscape surrounding a pollution source preclude housing at 
those locations. Examples will include coastal areas, areas where there are large parks, or urban areas with expanses of industrially or commercially zoned properties.

In sum, if both distance and direction matter to housing prices, the two variables are correlated, and direction is omitted, the coefficient on the distance variable can be expected to be biased. Ignoring direction while estimating a distance gradient can significantly compromise both the accuracy and the precision with which distance gradients are measured. This bias and loss in precision is amplified when directional effects are more prominent.

When direction is not part of the model, we have already noted that the spatial level curves of the $E\left[Y_{i}\right]$ are implicitly assumed to be circular. With directional diffusion of airborne pollutants, one would expect the contours of the dispersal pattern to be non-circular. Relaxing the implicit assumption of circular contour lines in the three-dimensional distance profile allows the researcher to estimate the direction of the main axis of a more general set of elliptical level curves. In the simple multiple-direction case illustrated in Figures 2 and 3 with data for four directions (North, South, East and West profiles), the implied three-dimensional distance profile will have the main axis of its elliptical level curves running from due West to due East. This is because of the assumption that the prevailing winds from due west. However, any appropriate empirical model needs sufficient flexibility to allow the orientation of the main axis of the elliptical contour lines to vary freely, based on spatial patterns in the dependent variable. Alternatively, one might constrain the main axis to correspond to the actual historical average wind direction. We now turn to viable specifications for such models. 


\section{$3 \quad$ Spatial trend models}

\subsection{Implications of a simple "planar spatial trend" model}

First, we show that the simplest linear-in-distance model with directional effects is equivalent to a model that includes just a simple linear distance effect plus a pair of incidental linear spatial trend variables. With GIS software, one can readily identify point locations of housing units or census tract centroids in decimal degrees (conventionally, to six decimal places). The simplest specification for a generic dependent variable $Y_{i}$ involves overlaying a conical direction-independent distance profile with some tilted plane defined over

longitude and latitude. ${ }^{3}$ The combination of the lat/long effects on the dependent variable and symmetric distance effects can readily mimic distance effects that are non-constant around the points of the compass:

$$
Y_{i}=\alpha+\beta d_{i}+\left(\gamma_{1} \operatorname{long}_{i}+\gamma_{2} \text { lat }_{i}\right)+\varepsilon_{i}
$$

Keep in mind that one degree of latitude is not the same distance as one degree of longitude. The length of one degree of longitude depends upon the latitude at which that distance is being calculated. In general one degree of longitude $=\cos \left(\right.$ latitude) ${ }^{*} 111.325$ kilometers. In contrast, one degree of latitude is well approximated by 110.6 kilometers. This complicates the task of determining the direction of steepest descent when longitude and latitude are used directly as explanatory variables. It is preferable to compute location in $(\mathrm{x}, \mathrm{y})$-space in common units in each direction.

Fortunately, it is not necessary to use the Greenwich Meridian and the equator as the origins of measurement for the absolute spatial location of each property in the sample. We recommend expressing both longitude and latitude in kilometers and shifting the origin of measurement to coincide with the site of the environmental disamenity. Denote the longitude and latitude of the site as $\left(x_{s}, y_{s}\right)$. Using the source of pollution as the origin of measurement, let $x_{i}$ be the east-west coordinate of the property, and let $y_{i}$ be the

\footnotetext{
${ }^{3}$ Any real specification will of course also control for a host of structural and neighborhood variables characteristics.
} 
north-south coordinate. Then

$$
Y_{i}=\alpha+\beta d_{i}+\left(\gamma_{1} x_{i}+\gamma_{2} y_{i}\right)+\varepsilon_{i}
$$

Here we assume that the longitude-to-kilometers conversion factor can be approximated for both the specific property and the pollution source by the latitude correction corresponding to their average latitude, so that

$$
\begin{aligned}
x_{i} & =(111.325)\left(\text { long }_{i}-\text { long }_{s}\right) \cos \left[\left(\text { lat }_{i}+\text { lat }_{s}\right) / 2\right] \\
y_{i} & =(110.6)\left(\text { lat }_{i}-\text { lat }_{s}\right)
\end{aligned}
$$

In equation (2), both the $x_{i}$ and $y_{i}$ distances are measured in kilometers, as is the distance $d_{i}{ }^{4}$ The parameters $\gamma_{1}$ and $\gamma_{2}$ in equation (2) will be different from their counterparts in equation (1) due to the change of location and scale.

It is convenient to convert equation (2) so that it is expressed entirely in terms of polar coordinates. Recall that $x_{i}=d_{i} \cos \theta_{i}$ and $y_{i}=d_{i} \sin \theta_{i}$ where $\theta_{i}$ is the direction from the site to housing unit $i$ (measured in radians counter-clockwise from due east). Making this substitution, equation (2) becomes:

$$
Y_{i}=\alpha+\beta d_{i}+\left(\gamma_{1} d_{i} \cos \theta_{i}+\gamma_{2} d_{i} \sin \theta_{i}\right)+\varepsilon_{i}
$$

Collecting the terms in distance, we get:

$$
Y_{i}=\alpha+\left(\beta+\gamma_{1} \cos \theta_{i}+\gamma_{2} \sin \theta_{i}\right) d_{i}+\varepsilon_{i}
$$

Instead of having a constant distance effect, the distance effect depends upon the direction in which it is being calculated.

\footnotetext{
${ }^{4}$ In implementing these transformations, it is crucial to remember that cartographers measure latitude in degrees from the equator, rather than in radians. The map measures of latitude must first be converted into the equivalent number of radians before using econometric software to calculate the cosine of the term in square brackets in the formulas in (3).
} 
One implication of this result is that researchers who have specified models with a linear spatial trend but no linear distance effect (i.e. $\beta=0$ ) are still estimating models with implicit direction and distance effects. However, the origin of measurement of these distances may be the intersection of the equator and the Greenwich Meridian, unless some other arbitrary point of origin is selected. More insidiously, any researcher who adds a set of linear spatial trend variables to a model that includes a specific distance measure may overlook the fact that the full distance effect is $\beta+\gamma_{1} \cos \theta_{i}+\gamma_{2} \sin \theta_{i}$, rather than simply $\beta$.

In describing fitted models involving heterogeneous parameters, it is customary to simplify the results by reporting key varying derivatives calculated at the "means of the data." While the average angle $\bar{\theta}$ in any sample will depend upon the spatial distribution of observations in that sample, it will usually be more convenient to use the fact that the average values of both $\cos \theta$ and $\sin \theta$ would be zero if this angle was uniformly distributed around the compass from 0 to $2 \pi$. The distance effect in this theoretical "average" direction corresponds to just the $\beta$ coefficient. If the researcher desires to know the predicted distance effect in each of the four main compass directions $(\mathrm{N}, \mathrm{W}, \mathrm{S}, \mathrm{E})$, these distance effects can be calculated as $\left(\beta+\gamma_{2}\right.$,

$\left.\beta-\gamma_{1}, \beta-\gamma_{2}, \beta+\gamma_{1}\right)$. The magnitudes of the directional distance effects thus clearly depend upon the signs and sizes of the two directional coefficients, $\gamma_{1}$ and $\gamma_{2}$.

\subsection{Implications of "quadratic spatial trend" specifications}

Some researchers control for systematic spatial trends by including quadratic and interaction terms in $x$ and $y$. (Consider the empirical example in Dubin (1998).) We show now that the combination of a fully quadratic spatial trend with a linear-in-distance specification leads to an implicitly quadratic-in-distance specification.

Let the model in conventional form be

$$
Y_{i}=\alpha+\beta d_{i}+\left(\gamma_{1} x_{i}+\gamma_{2} y_{i}+\gamma_{3} x_{i}^{2}+\gamma_{4} x_{i} y_{i}+\gamma_{5} y_{i}^{2}\right)+\varepsilon_{i}
$$

Converting this mixed Cartesian coordinate and distance specification into exclusively polar coordinates 
yields the following equivalent specification:

$$
\begin{aligned}
Y_{i}= & \alpha+\beta d_{i}+\left(\gamma_{1} d_{i} \cos \theta_{i}+\gamma_{2} d_{i} \sin \theta_{i}\right) \\
& +\left(\gamma_{3} d_{i}^{2} \cos ^{2} \theta_{i}+\gamma_{4} d_{i}^{2} \cos \theta_{i} \sin \theta_{i}+\gamma_{5} d_{i}^{2} \sin ^{2} \theta_{i}\right)+\varepsilon_{i}
\end{aligned}
$$

Simplify by collecting terms in $d_{i}$ and $d_{i}^{2}$ to reveal the equivalent form:

$$
\begin{aligned}
Y_{i}= & \alpha+\left(\beta+\gamma_{1} \cos \theta_{i}+\gamma_{2} \sin \theta_{i}\right) d_{i} \\
& +\left(\gamma_{3} \cos ^{2} \theta_{i}+\gamma_{4} \cos \theta_{i} \sin \theta_{i}+\gamma_{5} \sin ^{2} \theta_{i}\right) d_{i}^{2}+\varepsilon_{i}
\end{aligned}
$$

However, even if the observed directions $\theta_{i}$ were distributed uniformly around the compass, the expected distance effect will not, in this model, collapse to just $\beta$, as was the case for equation (5). The expected values of $\cos \theta_{i}, \sin \theta_{i}$ and $\cos \theta_{i} \sin \theta_{i}$ will all be zero in this case, but the expected values of $\cos ^{2} \theta_{i}$ and $\sin ^{2} \theta_{i}$ will not. We are considering the expected value over a uniform distribution for $\theta$ over the interval from 0 to $2 \pi$. This distribution has a constant density of $1 / 2 \pi$ that can be factored out of the integral in the expectation formulas:

$$
\begin{aligned}
& E\left[\cos ^{2} \theta\right]=\frac{1}{2 \pi} \int\left(\cos ^{2} \theta\right) \partial \theta=\left[\frac{1}{2} \theta-\frac{1}{4} \sin 2 \theta\right]_{0}^{2 \pi}=\frac{1}{2} \\
& E\left[\sin ^{2} \theta\right]=\frac{1}{2 \pi} \int\left(\sin ^{2} \theta\right) \partial \theta=\left[\frac{1}{2} \theta-\frac{1}{4} \sin 2 \theta\right]_{0}^{2 \pi}=\frac{1}{2}
\end{aligned}
$$

The expectations of these two terms will be strictly positive and both equal to 0.5 . Thus, for uniformly distributed directions, the specification in equation (8) corresponds to an average distance effect as follows:

$$
Y_{i}=\alpha+\beta d_{i}+\left(\frac{\gamma_{3}+\gamma_{5}}{2}\right) d_{i}^{2}+\varepsilon_{i}
$$


Clearly, even if we are considering the average distance effect over all possible directions, a model with both a simple linear distance effect and a full set of quadratic spatial trend terms actually implies a model with a quadratic distance effect. The average distance effect will be linear only if the average of the estimated coefficients on the squared $x$ and $y$ coordinates is not different from zero.

\subsection{Nonlinear-in-distance specifications}

Except for (i) changes of location and scale, (ii) the slight local approximation involving $\cos \left[\left(\right.\right.$ lat $\left.\left._{i}+l_{\text {lat }}\right) / 2\right]$, and (iii) conversion exclusively polar coordinates, the model in equation (5) is identical to that involving the simple planar spatial trend in terms of the longitude and latitude variables in equation (1). However, there is nothing to mandate using only the functional form employed in equations (1) or (2). In fact, this form has the unappealing characteristic that in any particular direction, the marginal effect of distance remains constant as distance increases. We generally expect the effect of localized externalities to diminish with distance until any incremental effect of distance essentially disappears.

To approximate a pattern of marginal distance effects that decrease with distance, researchers often use models that are nonlinear but monotonic transformations of the distance variable, $f\left(d_{i}\right)$. Candidate functional forms include a logarithmic transformation $f\left(d_{i}\right)=\ln \left(d_{i}\right)$, or a reciprocal function, $f\left(d_{i}\right)=1 / d_{i}$. However, both of these specifications can produce extreme values for the transformed distance variable as distance goes to zero. An easy fix for this problem is to shift the origin of measurement for the distance variable by adding one unit for all observations. Distance measurements are always non-negative, so this change-of-origin by one unit can guarantee that the smallest observed value of $f\left(d_{i}\right)=\ln \left(d_{i}+1\right)$ is zero and the largest observed value of $f\left(d_{i}\right)=1 /\left(d_{i}+1\right)$ is 1 . To keep the notation simple in what follows, we will refer to the distance variable generically, as $f\left(d_{i}\right)$. We adapt the model in equation (2) to allow for diminishing marginal effects of distance, but still allow the marginal effect at any given distance to vary systematically 
and smoothly with direction by substituting a suitable version of $f\left(d_{i}\right)$ for $d_{i}$ in our earlier model:

$$
Y_{i}=\alpha+\left(\beta+\gamma_{1} \cos \theta_{i}+\gamma_{2} \sin \theta_{i}\right) f\left(d_{i}\right)+\varepsilon_{i}
$$

To test statistically for the presence of directional heterogeneity in the distance effect, one would again test the joint hypothesis that $\gamma_{1}=\gamma_{2}=0$.

Over all possible directions either for the model in (1) and (2) or for the alternative model in equation (11), the two extrema of the distance effect occur where the derivative with respect to $\theta_{i}$ of the systematically varying coefficient, $\beta+\gamma_{1} \cos \theta_{i}+\gamma_{2} \sin \theta_{i}$, goes to zero. Making use of the facts that $\partial \sin \theta / \partial \theta=\cos \theta, \partial \cos \theta / \partial \theta=-\sin \theta$, and $\cos \theta / \sin \theta=\tan \theta$, the maximum predicted distance effect occurs at $\theta^{*}=\arctan \left(\gamma_{2} / \gamma_{1}\right)$, while the minimum distance effect lies at $\theta^{* *}=\theta^{*}+\pi$. If we are considering a hedonic property value model using equation (11), housing prices would be predicted to increase most slowly as one moves away from the site in the direction

$$
\theta^{* *}=\arctan \left(\gamma_{2} / \gamma_{1}\right)+\pi
$$

This direction depends upon the estimated parameters $\gamma_{1}$ and $\gamma_{2}$. This direction (measured in radians counterclockwise from due east) can be converted to compass degrees (measured clockwise from due north) by computing $\phi^{* *}=-180\left(\theta^{* *} / \pi\right)+90$. If the level curves of the property value distribution are non-circular only because of localized air pollution levels, this direction would be interpreted as the apparent downwind direction.

\subsection{Other patterns of directional heterogeneity}

Models with the slope coefficient on $f\left(d_{i}\right)$ generalized to simply $\beta+\gamma_{1} \cos \theta_{i}+\gamma_{2} \sin \theta_{i}$ do not capture the universe of all possible directional or spatial patterns in distance effects, although we will concentrate on 
these forms below. As section 3.2 suggests, a set of quadratic spatial trend variables can capture more general spatial patterns in the same family. These models may be appropriate in some empirical applications. Other functions of direction can also be explored, including variants that allow for systematic shifts in distance effects for subsets of directions according to the topography surrounding the source of pollution. Dummy variables for certain directions can be interacted with $\cos \theta_{i}$ and $\sin \theta_{i}$ to permit shifts in the directional effects away from the simple pattern captured by just $\beta+\gamma_{1} \cos \theta_{i}+\gamma_{2} \sin \theta_{i}$. Whenever physical features of the context may be important, their potential distinct effects should be explored.

Sometimes directional heterogeneity in distance effects will not vary around the compass relative to the source of the pollution as in the case of prevailing winds. There will be other cases when relative elevation, rather than simply compass direction, is the physically relevant determinant of distance effects. It may not be the angle relative to due east, but rather the angle relative to the downhill direction that matters most. If steepness in the downhill direction also matters, the trigonometric terms involving this angle will need to be interacted with the degree of slope in that downhill direction.

\section{Estimated versus actual "downwind" direction}

In this paper, we will use the term "downwind" to imply that prevailing winds constitute the physical process that leads to more-distant effects of pollution in one direction. However, we emphasize that the downwind terminology can also be generalized to imply all physical processes that contribute to spatially asymmetric transport of pollutants from a source, including the movement of surface or groundwater, and topographical effects on visual and noise polluation.

One potential problem with the unrestricted specification in equation (11) is that the estimated downwind direction, $\theta^{* *}=\arctan \left(\gamma_{2} / \gamma_{1}\right)+\pi$, may not correspond to the known downwind direction. The estimated downwind direction may be biased by other factors unrecognized by the researcher. To avoid such omitted variables bias, it will be important to control for other factors that can be expected to contribute to a general 
spatial price gradient.

Once the model in equation (11) has been estimated, therefore, it will be important to test whether the estimated "downwind" direction, $\phi^{* *}$ measured in compass degrees, coincides with the meteorological facts. ${ }^{5}$ This statistical test of the estimated wind direction would involve constructing a point estimate and standard error for the estimated direction $\theta^{* *}$ from the point estimates of parameters $\gamma_{1}$ and $\gamma_{2}$ and testing whether this direction could be equal to the actual downwind direction, $\theta^{0}$. Historical prevailing wind directions for major cities in the US are provided by NOAA (1998).

\subsection{Imposing the annual average prevailing wind direction}

In some cases, the downwind direction should not be estimated, but should be determined from meteorological data and imposed upon the model. Assume initially that the direction of prevailing winds is constant over the seasons. Let the actual downwind direction from the environmental disamenity be $\theta^{0}$ radians. If we wish to impose this downwind direction as a constraint on our estimation, it will translate into a restriction on the admissible values of $\gamma_{1}$ and $\gamma_{2}$. Solve equation (12) for the admissible relationship between $\gamma_{1}$ and $\gamma_{2}$ when $\theta^{* *}=\theta^{0}$ :

$$
\gamma_{2}=\gamma_{1} \tan \left(\theta^{0}+\pi\right)
$$

Substitute this restriction into equation (11) to yield

$$
\begin{aligned}
Y_{i} & =\alpha+\left(\beta+\gamma_{1} \cos \theta_{i}+\left[\gamma_{1} \tan \left(\theta^{0}+\pi\right)\right] \sin \theta_{i}\right) f\left(d_{i}\right)+\varepsilon_{i} \\
& =\alpha+\beta f\left(d_{i}\right)+\gamma_{1}\left[\cos \theta_{i}+\tan \left(\theta^{0}+\pi\right) \sin \theta_{i}\right] f\left(d_{i}\right)+\varepsilon_{i}
\end{aligned}
$$

This model can be estimated using conventional linear-in-parameters specifications since all terms inside the square brackets are observed data or known constants. This specification allows for directional asymmetry

\footnotetext{
${ }^{5}$ Recall that meteorologists report wind direction based on the direction from which the wind is coming, rather than the vector describing the direction in which it is blowing. Thus, a NW wind would be blowing "out of the NW, in a SE direction."
} 
in the distance effect, but admits for distortions only in a direction known to be consistent with prevailing winds. To test whether there is evidence of a directional effect in the dependent variable that coincides with wind direction (or other natural flows that may affect waterborne contaminants, for example), one would simply test whether $\gamma_{1}=0$ can be rejected.

We might desire this directional restriction on the downwind effect because without it, there is no requirement that the direction in which housing prices increase most slowly with distance actually coincides with the direction in which pollution travels the farthest. Allowing the distance profile implied by the model to "tilt" in any arbitrary direction will court omitted variables bias. There may be other underlying factors, unknown to the researcher, which account for an overarching spatial trend in housing prices. These factors could include a temperature gradient, distance from a nearby city center or coastline, or any other amenity or disamenity that is not controlled for by its explicit inclusion in the specification.

In some applications, however, we may wish to isolate the extent of the downwind effect separately from any additional nonspecific directional effect that may be superimposed upon the downwind effect. We can introduce parameters $\gamma_{1}^{*}$ and $\gamma_{2}^{*}$ and allow for two distinct types of directional effects:

$$
\begin{gathered}
Y_{i}=\alpha+\beta f\left(d_{i}\right)+\gamma_{1}\left[\cos \theta_{i}+\tan \left(\theta^{0}+\pi\right) \sin \theta_{i}\right] f\left(d_{i}\right) \\
+\left(\gamma_{1}^{*} \cos \theta_{i}+\gamma_{2}^{*} \sin \theta_{i}\right) f\left(d_{i}\right)+\varepsilon_{i}
\end{gathered}
$$

The constructed variable $\left[\cos \theta_{i}+\tan \left(\theta^{0}+\pi\right) \sin \theta_{i}\right] f\left(d_{i}\right)$ will vary independently from $\cos \theta_{i} f\left(d_{i}\right)$ and $\sin \theta_{i} f\left(d_{i}\right)$, so that distinct estimates can be obtained for the downwind effect parameter, $\gamma_{1}$, and the generic directional effect parameters, $\gamma_{1}^{*}$ and $\gamma_{2}^{*}$. The researcher will need to speculate upon logical explanations for statistical significance in these two additional parameters. ${ }^{6}$

\footnotetext{
${ }^{6}$ Joe Stone suggested this potentially useful generalization.
} 


\subsection{Imposing seasonally varying prevailing winds}

A more intricate model may be appropriate when there are regular seasonal differences in the direction of prevailing winds. The term in equation (14) that carries the $\gamma_{1}$ coefficient captures the direction, $\theta_{i}$, from the localized environmental disamenity which will be constant over time but will vary across observations. This term also includes the direction of prevailing winds, $\theta^{0}$. This is consistent with the assumption that the direction of the prevailing winds is fixed across observations. However, this assumption may be unrealistic. For example, selling prices of houses are understood to vary seasonally for a variety of reasons. If they also vary seasonally as a result of some seasonal pattern of dispersion of some point source pollutant, this information can also be employed to enhance estimation if time-subscripted data are available. The model could be generalized to apply to $Y_{i t}$ and $\theta_{t}^{0}$ :

$$
Y_{i t}=\alpha+\beta f\left(d_{i}\right)+\gamma_{1}\left[\cos \theta_{i}+\tan \left(\theta_{t}^{0}+\pi\right) \sin \theta_{i}\right] f\left(d_{i}\right)+\varepsilon_{i t}
$$

As before, $\theta_{t}^{0}$ is not a parameter to be estimated, but additional data on seasonal wind directions to be employed in the estimation process.

\section{Level curves of distance profiles}

For any of these directional models, it may be also be useful to derive the implied level curves for the overall distance profile. Once the unknown parameters of the model have been estimated, one can solve for the latitude and longitude coordinates of locations that lie along level curves for fitted $Y_{i}$. The geo-coded level curves can then be displayed using mapping software. For the basic model in equation (11), implementation

proceeds as follows. Assume $\varepsilon_{i}=0$ and solve the fitted model for the values of $d_{i}^{*}$ that correspond to each of the observed directions $\left(\theta_{i}\right)$ represented in the sample if $Y_{i}$ is held constant at $\mathrm{Y}^{*}$. The set of polar 
coordinates satisfying this condition will be:

$$
\left[d_{i}^{*}, \theta_{i}\right]=\left[f^{-1}\left(\frac{Y^{*}-\alpha}{\beta+\gamma_{1} \cos \theta_{i}+\gamma_{2} \sin \theta_{i}}\right), \theta_{i}\right]
$$

Now convert these points expressed in terms of polar coordinates back into Cartesian coordinates using

$$
\begin{aligned}
& x_{i}=d_{i}^{*} \cos \theta_{i} \\
& y_{i}=d_{i}^{*} \sin \theta_{i}
\end{aligned}
$$

Then convert these simple Cartesian coordinate back into latitude and longitude by reversing the transformations in (3):

$$
\begin{aligned}
\text { lat }_{i}^{*} & =\text { lat }_{s}+\left(y_{i}^{*} / 110.6\right) \\
\text { long }_{i}^{*} & =\text { long }_{s}+x_{i}^{*} /\left[111.325 \cos \left[\left(\text { lat }_{i}^{*}+\text { lat }_{s}\right) / 2\right]\right]
\end{aligned}
$$

If the observations are first sorted in order of $\theta_{i}$, the graphing routines in conventional estimation software can be used to connect the points and draw a smooth curve. Saving the latitudes and longitudes and mapping these pairs of points will produce an elliptical pattern of points. Alternatively, to produce an ellipse consisting of an arbitrarily dense pattern of points, one could discard the observed directions, $\theta_{i}$, simulate as many evenly spaced values as desired between 0 and $2 \pi$ and perform the transformations in equations (17) through (19) using these simulated values instead.

\section{Hedonic models and optimal source reduction}

In this section, we outline how hedonic property value models, with or without directional heterogeneity in distance effects, can be integrated into models for optimal pollution abatement when there is spatial 
heterogeneity in non-uniformly-mixing pollutants emitted by a number of point sources.

The usual model for non-uniformly mixing pollutants assumes that the ambient concentration at receptor $i$ depends upon emissions from source $j$, where ambient environmental quality at $i$ is given by the transport function $T_{j i}\left(e_{i}\right)$ (see Helfand, Berck and Maull (2003), p. 264). If we assume that damages at location $i$ are due to the simple aggregate of emissions reaching that place, then damages at that location are $D_{i}\left(\sum_{j=1}^{K} T_{j i}\left(e_{i}\right)\right)$. A simpler assumption is that the transport function is linear and additively separable in emissions from each source (Kolstad, 2000, p. 156). Then the relationship between emissions and ambient concentrations can be summarized by the appropriate element $a_{i j}$ of the "transfer matrix," A. Assume that the ambient concentration in parts per million of the pollutant in question at receptor $i, p p m_{i}$, depends on emissions levels $e_{i}$ from each source $j=1, \ldots, K$ according to this approximately linear relationship:

$$
\operatorname{ppm}_{i}=a_{0}+a_{i 1} e_{1}+\ldots+a_{i K} e_{K}+u_{i}, \quad i=1, \ldots, N
$$

where $u_{i}$ is an error term and the transfer coefficients $a_{i j}$ are measured in parts-per-million of ambient concentration at receptor $i$ per ton of pollution emitted at source $j$. Efficient abatement at each of the $j=1, \ldots, K$ different sources requires that the decision-maker adjust emission levels from all sources to maximize net social benefits (the difference between overall social benefits of reduced emissions and the overall costs to firms of abatement).

The economic theory of optimal abatement under these circumstances is relatively mature, but policy implementation is hampered in many cases by a paucity of data on the relevant transport functions. We will now outline how hedonic property value models can be used to quantify the product of marginal ambient damages and transport function derivatives. In certain cases, this product of terms is all the transport information that is needed, so separate quantification of the transport function may not be necessary. Socially optimal allocations of abatement responsibility can be informed by hedonic property value models. 


\subsection{Housing units as implicit "ambient receptor sites"}

Suppose that the type of environmental disamenity being considered is an example of a "localized externality" according to the distinctions drawn in Palmquist (1992) and Palmquist (2003). This means that the environmental disamenity affects only a small number of properties relative to the size of the market. We will also assume that there are no significant transactions or moving costs. Under these conditions, first-stage hedonic property value estimates are appropriate for welfare calculations. Hedonic rental rate differentials for localized externalities can be integrated into our conventional models that articulate the equimarginal principle for optimal abatement or emissions at a finite number of different pollution sources.

\subsubsection{Marginal social costs of pollution (marginal benefits of abatement)}

Suppose the sum across properties of the individual decrements in implicit housing rental rates are a reasonable approximation to the social benefits of eliminating pollution. For each of the housing units in the estimating sample, the individual social benefit of reducing pollution by $\Delta e_{j}$ at each source is given by:

$$
\begin{aligned}
\Delta \text { rental rate }_{i} & =\frac{M S C_{i}}{\Delta p p m_{i}} \sum_{j=1}^{K}\left(\frac{\Delta p p m_{i}}{\Delta e_{j}}\right) \Delta e_{j} \\
& =\frac{M S C_{i}}{\Delta p p m_{i}} \sum_{j=1}^{K} a_{i j} \Delta e_{j}
\end{aligned}
$$

This assumes that the rental rate differential for a property is the sum of the differentials due to the portion of ambient pollution at receptor $i$ attributable to each of the $K$ different sources.

Assume that the dependent variable in a typical hedonic property value model is the annualized rental rate, $Z_{i}$, that corresponds to the selling price of the property, $Y_{i}$. Adapting our simple model of equation (11) to the case of multiple sources of pollution, we can specify this rental rate as a function of the distances and directions of the property relative to several point sources of pollution. Assume for now that all properties are otherwise identical, in terms of size as well as structural and neighborhood characteristics, so that there is no need to control for other sources of heterogeneity besides distance and direction from the various pollution 
sources. A specification for the annualized rental rate function with multiple localized pollution sources, $j=1, \ldots, K$ could be:

$$
Z_{i}=\alpha+\sum_{j=1}^{K}\left(\beta_{j}+\gamma_{j 1} \cos \theta_{j i}+\gamma_{j 2} \sin \theta_{j i}\right) f_{j}\left(d_{j i}\right)+\varepsilon_{i}
$$

The compound coefficients $\left(\beta_{j}+\gamma_{j 1} \cos \theta_{j i}+\gamma_{j 2} \sin \theta_{j i}\right)$ in equation (22) are measured in dollars per unit of distance and the specification assumes implicitly that the quantity and type of pollutant emitted from each source is identical. To allow for different transformations of distance to apply for each pollution source, we now index $f\left(d_{j i}\right)$ by $j$ to create $f_{j}\left(d_{j i}\right)$, although for similar pollutants, the identical transformation may apply to distances from all sources. Suppose now that the distance effects are estimated per unit of pollutant emitted, so that the overall impact of the total emissions from source $j$ will be this marginal effect times the level of emissions from that source, $e_{j}$. The model in (22) can be generalized to the case of varying levels of emissions from each source:

$$
Z_{i}=\alpha+\sum_{j=1}^{K}\left(\beta_{j}+\gamma_{j 1} \cos \theta_{j i}+\gamma_{j 2} \sin \theta_{j i}\right) f_{j}\left(d_{j i}\right) e_{j}+\varepsilon_{i}
$$

With this modification, the change in the implicit rental rate of the $i^{t h}$ property with respect to a change in the emissions from the $j^{\text {th }}$ pollution source is a function of the distance between that source and the property in question. Viewing this specification from the perspective of equation (21), we can identify the correspondence

$$
\frac{\Delta \text { rental rate }_{i}}{\Delta e_{j}}=\frac{M S C_{i}}{\Delta p p m_{i}} a_{i j}=\left(\beta_{j}+\gamma_{j 1} \cos \theta_{j i}+\gamma_{j 2} \sin \theta_{j i}\right) f_{j}\left(d_{j i}\right)
$$

Thus, the effect of a unit of emissions from the $j^{\text {th }}$ pollution source on the implicit rental rate of the $i^{\text {th }}$ property subsumes both the marginal social cost to that property of the $j^{\text {th }}$ source's contribution to ambient 
concentrations and the relevant transfer coefficient.

The overall social benefit of a vector of pollution reductions at the set of $K$ sources will be the sum of these individual effects across all $N$ housing units in the affected area (not just the $N^{*}$ units in the estimating sample from which the hedonic distance coefficients are derived). The overall marginal social benefits of emissions reductions $\left(\Delta e_{1}, \ldots, \Delta e_{K}\right)$ will be equal to the overall marginal social costs $(M S C)$ that this pattern of pollution created:

$$
M S C=\sum_{i=1}^{N}\left(\sum_{j=1}^{K}\left(\beta_{j}+\gamma_{j 1} \cos \theta_{j i}+\gamma_{j 2} \sin \theta_{j i}\right) f_{j}\left(d_{j i}\right) \Delta e_{j}\right)
$$

\subsubsection{Marginal social benefits of pollution (marginal costs of abatement)}

The marginal costs of pollution abatement to the industry are the same as the marginal savings to the industry from continuing to pollute. The aggregate marginal benefits to the $K$ firms of the incremental changes in emissions summarized as $\left(\Delta e_{1}, \ldots, \Delta e_{K}\right)$ will be:

$$
M S=\sum_{j=1}^{K} \operatorname{mac}_{j} \Delta e_{j}
$$

where $m a c_{j}$ is the marginal abatement cost (marginal cost of emissions reduction) for source $j$. Suppose abatement costs are a simple quadratic function of abatement level $b_{j}$ with no intercept or linear term: $a c_{j}=\delta_{j} b_{j}^{2}$. Then marginal abatement costs will be proportional to abatement levels: $\operatorname{mac}_{j}=2 \delta_{j} b_{j}=c_{j} b_{j}$.

\subsubsection{Efficient abatement}

Equalizing overall marginal social costs and overall marginal social benefits can be accomplished by equalizing the marginal social costs and marginal social benefits associated with emissions from each source:

$$
\left[\sum_{i=1}^{N}\left(\beta_{j}+\gamma_{j 1} \cos \theta_{j i}+\gamma_{j 2} \sin \theta_{j i}\right) f_{j}\left(d_{j i}\right)\right] \Delta e_{j}=\left[c_{j} b_{j}\right] \Delta e_{j} \quad j=1, \ldots, K
$$


The term $\Delta e_{j}$ cancels from each side of this equimarginal condition and we are left with the result that the optimal level of abatement $b_{j}$ for each firm will be determined by:

$$
b_{j}=\left(1 / c_{j}\right) \sum_{i=1}^{N}\left(\beta_{j}+\gamma_{j 1} \cos \theta_{j i}+\gamma_{j 2} \sin \theta_{j i}\right) f_{j}\left(d_{j i}\right)
$$

There is still more information that can be brought to bear on the estimation process and the determination of the socially optimal level of emissions reduction for each source. If the pollutant is dispersed by prevailing winds, we can impose a dispersal pattern consistent with those prevailing winds. If we adapt equation (23) to the case with constant prevailing winds, as in equation (14), we get

$$
Z_{i}=\alpha+\sum_{j=1}^{K}\left[\beta_{j}+\gamma_{j 1}\left(\cos \theta_{i}+\tan \left(\theta_{t}^{0}+\pi\right) \sin \theta_{i}\right)\right] f_{j}\left(d_{j i}\right) e_{j}+\varepsilon_{i}
$$

If the effect of distance and direction on the per-unit effects of emissions is the same across all sources, so that $\beta_{j}=\beta$ and $\gamma_{j 1}=\gamma_{1}$ for all $j$, a resulting special case of this hedonic model would be:

$$
Z_{i}=\alpha+\beta \sum_{j=1}^{K} f_{j}\left(d_{j i}\right) e_{j}+\gamma_{1} \sum_{j=1}^{K}\left(\cos \theta_{i}+\tan \left(\theta_{t}^{0}+\pi\right) \sin \theta_{i}\right) f_{j}\left(d_{j i}\right) e_{j}+\varepsilon_{i}
$$

If the hypotheses that $\beta_{j}=\beta$ and $\gamma_{j 1}=\gamma_{1}$ for all $j$ cannot be rejected, then each unit of pollutant from any localized source has the same effect on the rental rates for any housing unit at the same distance and in the same direction.

With quadratic abatement costs and the estimated downwind direction constrained to match the empirical downwind direction based on typical weather patterns, the socially optimal level of abatement for each source 
can be determined as follows:

$$
\begin{aligned}
b_{j} & =\left(1 / c_{j}\right) \sum_{i=1}^{N}\left[\beta_{j}+\gamma_{j 1}\left(\cos \theta_{i}+\tan \left(\theta_{t}^{0}+\pi\right) \sin \theta_{i}\right)\right] f_{j}\left(d_{j i}\right) \\
& =\left(\beta_{j} / c_{j}\right) \sum_{i=1}^{N} f_{j}\left(d_{j i}\right)+\left(\gamma_{j 1} / c_{j}\right) \sum_{i=1}^{N}\left[\cos \theta_{i}+\tan \left(\theta_{t}^{0}+\pi\right) \sin \theta_{i}\right] f_{j}\left(d_{j i}\right)
\end{aligned}
$$

If we constrain the per-unit effects of pollutants from each source to be identical at the same distance and in the same direction as in equation (30), the equation in (31) can be simplified. If all firms also face identical cost structures, so that the slopes of their marginal cost curves are identical and $c_{j}=c$ for all sources $j$, then the socially optimal level of abatement for each source will be:

$$
b_{j}=(\beta / c) \sum_{i=1}^{N} f_{j}\left(d_{j i}\right)+\left(\gamma_{1} / c\right) \sum_{i=1}^{N}\left[\cos \theta_{i}+\tan \left(\theta_{t}^{0}+\pi\right) \sin \theta_{i}\right] f_{j}\left(d_{j i}\right)
$$

In words, even if abatement technology is identical for all firms, the spatial distribution of housing units relative to each site will imply different optimal levels of emissions $b_{j}$ for each firm. As expected, these optimal abatement levels will depend on the location of houses relative to each firm and on the direction of the prevailing winds.

If there is no directional heterogeneity in any of the distance effects for any of the individual pollution sources, then $\gamma_{1}=0$ and equation (33) reduces to just

$$
b_{j}=\frac{\beta}{c} \sum_{i=1}^{N} f_{j}\left(d_{j i}\right)=\frac{N \beta}{c} \overline{f_{j}\left(d_{j i}\right)}
$$

This form implies that only the number of affected housing units and the average of the relevant function of distance matters, along with the common distance effect $(\beta)$ and the common marginal abatement cost parameter, $c$. If $f_{j}\left(d_{j i}\right)=d_{j i}$ for all sources $j$, then simply the average distance will enter the formula.

If abatement costs are approximately quadratic and the (constant) slope of the marginal abatement 
cost function for each source is known, then equation (31), in conjunction with locational polar-coordinate data for all affected housing units, $\left(d_{j i}, \theta_{j i}\right)$ relative to each source $j$, combined with information about the downwind direction for prevailing winds, $\theta^{0}$, allows a tedious but straightforward calculation of the socially optimal level of abatement for each source.

\section{Caveats and Directions for Future Research}

Researchers who explore directional heterogeneity in distance effects will still need to be vigilant about the possibility of omitted variables biases in their estimated distance effects. Collinearity among distances to different pollution sources, and to other amenities and disamenities that can also influence housing prices, will need to be assessed. One important class of sensitivity tests might include the demonstration that similar functional forms for distance and direction, computed relative to some randomly selected point (or points), exhibits no such directional distance effects on housing prices. ${ }^{7}$

Appropriate specifications for the form of directional heterogeneity in distance effects should typically refer to the physical features of the application (including prevailing winds, direction of groundwater movement or surface-water flow, and/or topography). It will be unwise to pursue any serious model without acquiring some knowledge of these processes or spatial features.

It is worth noting that nothing about the specification in equation (11) requires that the fitted distance effect be positive in all directions. If the housing price differentials captured by the distance effect are entirely due to the localized environmental disamenity, one would expect that the distance effect should be strictly positive in all directions. This hypothesis can be tested. If the distance effect is negative in some directions, this will call into question the assumption that distance is exclusively a proxy for ambient environmental quality. Negative distance effects in some directions may be a consequence of other omitted spatial variables. Of course, even when the estimated distance effect is positive in all directions, omitted spatial variables can

\footnotetext{
${ }^{7}$ Richard Ready contributed this suggestion.
} 
still cause mischief, but the presence of some negative distance effects should certainly raise suspicion about the completeness of the specification.

If the researcher is willing to resort to non-linear-in parameters specifications, the sign of the distance effect could be constrained to be positive in all directions by estimating the logarithm of the distance effect, rather than its level. This corresponds to using the specification:

$$
Y_{i}=\alpha+\left[\exp \left(\beta+\gamma_{1} \cos \theta_{i}+\gamma_{2} \sin \theta_{i}\right)\right] f\left(d_{i}\right)+\varepsilon_{i}
$$

Here, the logarithm of the distance effect varies systematically with direction.

If the environmental externality in question is not a localized externality, so that the hedonic price schedule is shifted for the entire market, then the first-stage hedonic equation is insufficient as a measure of the loss in social welfare due to pollution. Richer welfare models will be necessary and the simple analysis in Section 6 pertaining to optimal levels of emissions for different firms will be inappropriate. For localized externalities, however, the method proposed here may be entirely suitable.

The data requirements for assessing the optimal levels of emissions for different point sources of localized pollution will be substantial, since it is necessary to approximate the locations of all affected properties, as well as to estimate the effect of the localized externality on each property in a sample of housing transactions prices. It will also be necessary to convert transactions prices into equivalent annualized rental rates, and to quantify the annual emissions from each plant and the marginal abatement costs for each plant. However, any formal benefit-cost analysis will typically require a lot of data.

All of the models developed in this paper pertain to just the systematic portion of a first-stage hedonic property value model, rather than the error terms. All of these generalizations will be amenable to being combined with state-of-the-art spatial error models, such as correlogram- or semivariogram-based models or lattice models (see the summary of methods provided by Pace et al. (1998)). 


\section{Conclusions}

The significant contribution in this paper is the development of a new empirical specification for spatial data concerning variables that may be affected by proximity to environmental hazards. We introduce direction as a potentially important determinant of distance (proximity) effects. We include a special case of this empirical model that can accommodate additional data about the direction of prevailing winds or other systematic physical features or processes. This special case involves a restriction on the parameter estimates that can still be accommodated within a conventional linear-in-parameters estimation framework. If there are seasonal patterns in prevailing winds or other processes that may lead to seasonal differences in the spatial pattern of the level of the disamenity from the environmental hazard, these data can also be exploited.

We have developed these new specifications as generally as possible. In the context of hedonic property value models, the dependent variable will typically be data on individual house selling prices. If environmental justice is the issue in question, however, the dependent variable could be the proportion of the population in different sociodemographic groups at different distances (and directions) from the localized environmental externality or externalities.

If direction matters but is ignored, the best-case result is that the researcher is limited to measuring the average distance effect, around the compass, with lesser precision than would be possible in a directionally heterogeneous model. A worst-case result is that the geographic distribution of observations is systematically correlated with direction (an omitted variable) so that the estimated distance effects are both biased and less precise. The implication and insight of this analysis is that prior research that has ignored substantial directional effects may have failed to identify statistically significant distance effects or may have presented biased results.

Finally, we have explored the possibility of interpreting the housing units in a hedonic property value study as individual ambient receptor sites. This allows hedonic property value models to be integrated with notions of socially optimal pollution abatement among multiple sources of localized pollution externalities. 
The insight drawn from this model is that optimal pollution levels for locally polluting firms emitting nonuniformly mixing pollutants can depend on more than just the marginal abatement costs of each firm. The distribution of housing units relative to each pollution source will also figure prominently in the calculations. If independently calibrated transport functions are not available for calculations of optimal abatement patterns, there is scope for using hedonic property value models to fill this gap. 


\section{REFERENCES}

Bartik, T.J., Smith, V.K. (1987). "Urban amenities and public policy". In E.S. Mills (ed.) Handbook of regional and urban economics. Amsterdam: North-Holland.

Dale, L., Murdoch, J.C., Thayer, M.A., Waddell, P.A. (1999). "Do property values rebound from environmental stigmas? Evidence from Dallas", Land Economics 75, 311-326.

Dubin, R.A. (1998). "Predicting house prices using multiple listings data", Journal of Real Estate Finance and Economics 17, 35-59.

Farber, S. (1998) "Undesirable facilities and property values: a summary of empirical studies," Ecological Economics, 24, 1-14.

Freeman, A.M.I. (1993). The measurement of environmental and resource values. Washington, DC: Resources for the Future.

Gillen, K., Thibodeau, T., Wachter, S. (2001). "Anisotropic autocorrelation in house prices", Journal of Real Estate Finance and Economics 23, 5-30.

Helfand, G.E., Berck, P., Maull, T. (2003) "The theory of pollution policy" Chapter 6 in Maler, K-G., Vincent, J.R. (eds.) Handbook of Environmental Economics, Volume 1: Environmental Degradation and Institutional Responses, Amsterdam: Elsevier.

Hite, D., Chern, W. Hitzhusen, F. Randall, A. (2001). "Property-value impacts of an environmental disamenity: the case of landfills", Journal of Real Estate Finance and Economics 22, 185-202.

Kiel, K., Zabel, J. (2001). "Estimating the economic benefits of cleaning up Superfund sites: The case of Woburn, Massachusetts", Journal of Real Estate Finance and Economics 22, 163-184.

Kiel, K.A. (1995). "Measuring the impact of the discovery and cleaning of identified hazardous-waste sites on house values", Land Economics 71, 428-435.

Kiel, K.A., McClain, K.T. (1995). "The effect of an incinerator siting on housing appreciation rates", Journal of Urban Economics 37, 311-323. 
Kohlhase, J.E. (1991). "The impact of toxic-waste sites on housing values", Journal of Urban Economics $30,1-26$.

Kolstad, C.D. (2000) Environmental Economics, New York: Oxford University Press

McMillen, D.P., Thorsnes, P. (2003). "The aroma of Tacoma: Time varying average derivatives and the effect of a superfund site on house prices", Journal of Business and Economic Statistics 21, 237-246.

Michaels, R.G., Smith, V.K. (1990). "Market-segmentation and valuing amenities with hedonic models - the case of hazardous-waste sites", Journal of Urban Economics 28, 223-242.

Pace, R.K., Barry, R., Sirmans, C.F. (1998). "Spatial Statistics and Real Estate," Journal of Real Estate Finance and Economics, 17, 5-13.

Palmquist, R.B. (1991). "Hedonic methods". In J.B. Braden, C.D. Kolstad (eds.), Measuring the demand for environmental quality. Amsterdam: North Holland.

Palmquist, R.B. (1992). "Valuing localized externalities", Journal of Urban Economics 31, 59-68.

Palmquist, R.B. (2003). Property value models. Department of Economics, North Carolina State University. Raleigh, NC. 85 .

Palmquist, R.B., Roka, F.M., Vukina, T. (1997). "Hog operations, environmental effects, and residential property values", Land Economics 73, 114-124. 


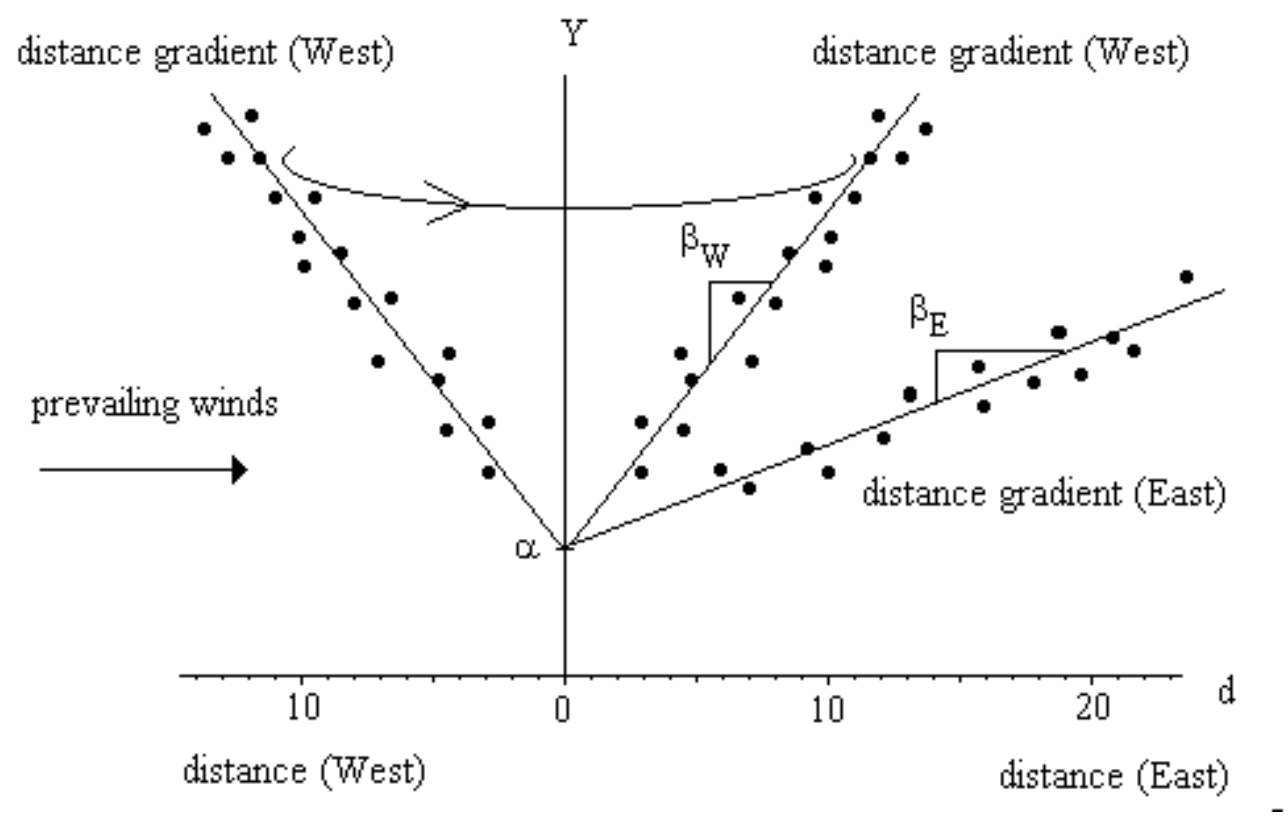

Figure 1: Bi-directional distance effects and imprecision in estimates 


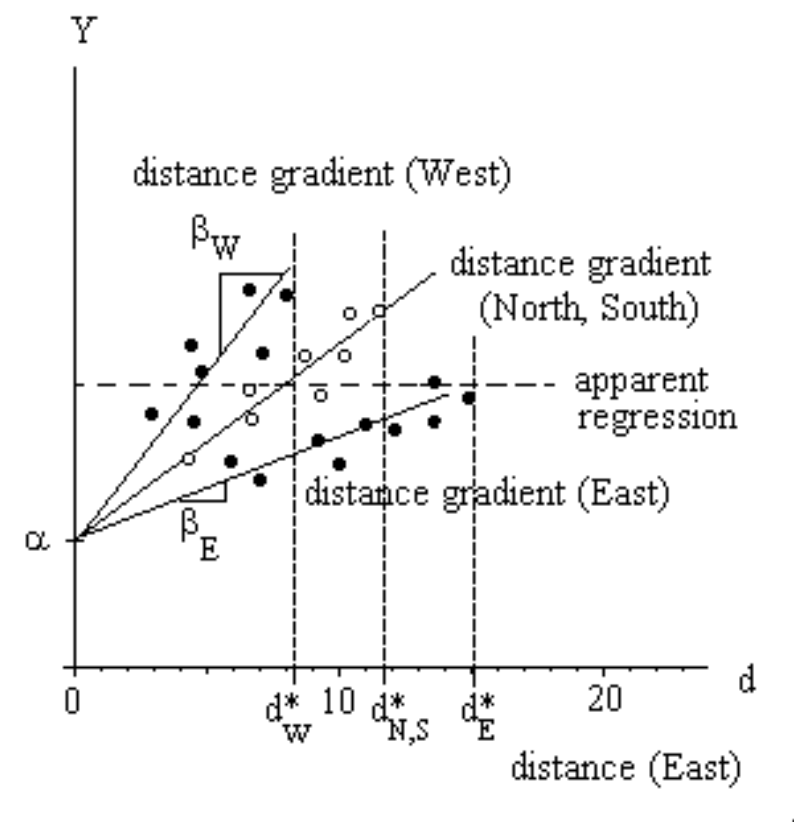

Figure 2: Four-directional distance effects and one possible source of bias

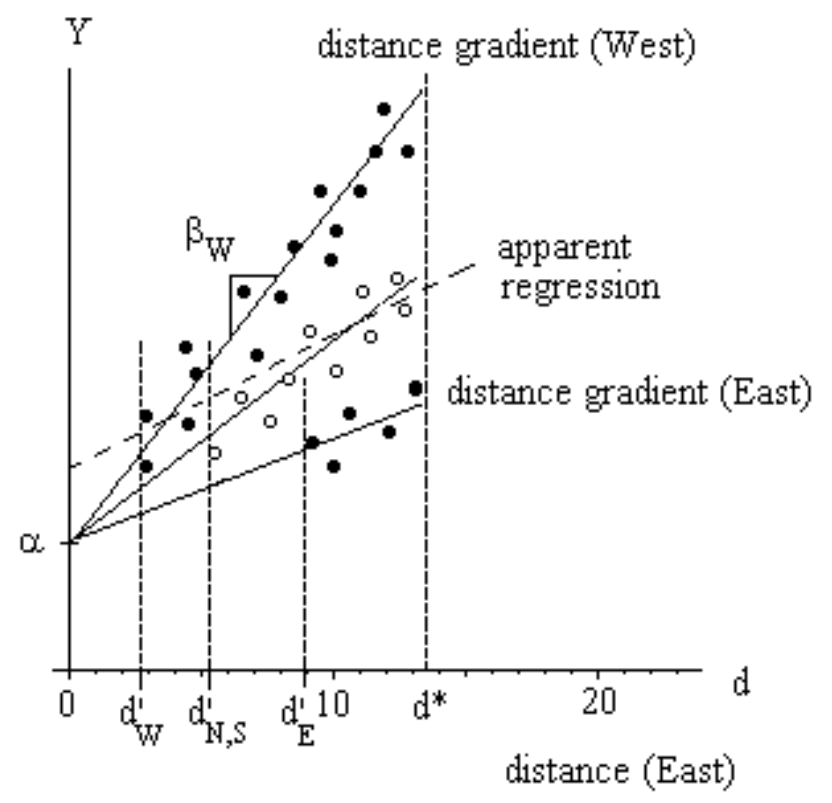

Figure 3: Four-directional distance effects and a second possible source of bias 